\title{
Probiotic Prescription Status of Pediatric Patients with Otitis Media Receiving Oral Amoxicillin or Amoxicillin/Clavulanate from April 2016 to March 2017 Using a Japanese Health Insurance Claims Database
}

\author{
Shungo Imai, ${ }^{a}$ Kenji Momo, ${ }^{b}$ Hitoshi Kashiwagi, ${ }^{a}$ Takayuki Miyai, ${ }^{c}$ Mitsuru Sugawara,${ }^{a, d}$ and \\ Yoh Takekuma*,d \\ ${ }^{a}$ Faculty of Pharmaceutical Sciences, Hokkaido University; Kita 12, Nishi 6, Kita-ku, Sapporo 060-0812, Japan: \\ ${ }^{b}$ Department of Hospital Pharmaceutics, School of Pharmacy, Showa University; 1-5-8 Hatanodai, Shinagawa-ku, \\ Tokyo 142-8555, Japan: ${ }^{c}$ Graduate School of Life Science, Hokkaido University; Kita 10, Nishi 8, Kita-ku, Sapporo \\ 060-0810, Japan: and ${ }^{d}$ Department of Pharmacy, Hokkaido University Hospital; Kita 14, Nishi 5, Kita-ku, Sapporo \\ 060-8648, Japan.
}

Received March 6, 2020; accepted December 11, 2020

Antibiotic-associated diarrhea (AAD) is a typical side effect of antibiotic treatment, especially in children. Amoxicillin (AMPC) and amoxicillin/clavulanate (AMPC/CVA) are associated with high risk of AAD; however, these antibiotics are important in the pediatric field. Recent research suggests that probiotics prevent pediatric AAD, including that caused by AMPC and AMPC/CVA. Indeed, guidelines for acute otitis media in children recommend the concomitant use of probiotics. However, the prescription status of probiotics for pediatric patients with otitis media receiving oral AMPC and AMPC/CVA remains unknown. We therefore conducted a survey to clarify the current status of these prescriptions and, in particular, to identify specific populations with a low proportion of probiotic prescriptions. Pediatric patients ( $\leq 15$ years of age) newly prescribed oral AMPC or AMPC/CVA for otitis media between April 2016 and March 2017 were identified from a Japanese health insurance claims database. Eligible patients were divided into the AMPC (1303 patients) and AMPC/CVA (424 patients) groups, in which $659(50.6 \%)$ and 293 (69.1\%) patients were prescribed probiotics, respectively. Of the patients receiving probiotic prescriptions in the AMPC and AMPC/CVA groups, $632(95.9 \%)$ and $286(97.6 \%)$ patients received antibiotic-resistant probiotic prescriptions, respectively. When classified by the prescribing clinical department and patient age, the proportions of probiotic prescriptions in Internal Medicine and Pediatrics departments were lower than those in the Otorhinolaryngology department regardless of age. These results indicate the probability of insufficient probiotic prescriptions for pediatric patients with otitis media. Solving this issue may lead to the provision of safer antimicrobial therapy.

Key words antibiotic-associated diarrhea; probiotics; insurance claim; oral antibiotics; pediatric patient

\section{INTRODUCTION}

Diarrhea, a typical side effect of antibiotics, occurs when the colonization potential of the gastrointestinal flora is disturbed. ${ }^{1)}$ Antibiotic-associated diarrhea (AAD) is often induced by an overgrowth of Clostridium difficile (C. difficile). ${ }^{1)}$ C. difficile-associated diarrhea is connected to serious symptoms and is often observed in elderly patients, immunocompromised patients, hospitalized patients, and children. ${ }^{2)}$

Oral antibiotics are often administered to pediatric patients; for example, for the treatment of respiratory infections. ${ }^{3)}$ The proportion of pediatric outpatients who suffer from AAD has been reported to range from 11 to $40 \%{ }^{4,5)}$ Amoxicillin (AMPC) and amoxicillin/clavulanate (AMPC/CVA) are considered to be associated with a high risk of AAD. ${ }^{4,6-9)}$ For example, in a meta-analysis targeting pediatric patients with otitis media, the occurrence of diarrhea was highest in children receiving high-dose AMPC/CVA. ${ }^{9)}$ However, they are important antibiotics in the pediatric field. ${ }^{10-13)}$ Indeed, in acute otitis media and respiratory tract infections, either AMPC or AMPC/CVA is recommended as the first-line treatment. ${ }^{10-12)}$ Thus, the prevention of AAD, especially in pediatric patients treated with AMPC and AMPC/CVA, is crucial.
Probiotics are defined as "live microorganisms, which, when administered in adequate amounts, confer a health benefit to the host." ${ }^{\text {"14) }}$ The effectiveness of probiotics for the prevention of pediatric AAD, especially those caused by AMPC and/or AMPC/CVA, has been established in recent reviews. ${ }^{15)}$ Meanwhile, many probiotics are inactivated by antimicrobials; thus, the use of antibiotic resistant probiotics may prove meaningful. ${ }^{16-18)}$ Indeed, guidelines for acute otitis media in children recommend the concomitant use of probiotics with antibiotic-resistant lactic acid bacteria preparations (RLABP) or C. butyricum preparations. ${ }^{12)}$ Therefore, the prophylactic administration of probiotics, especially those with antibiotic resistance, may be effective in pediatric patients with otitis media who receive AMPC and AMPC/CVA.

However, the prescription status of probiotics for children receiving these antibiotics is largely unknown. In particular, the identification of specific populations with low proportions of probiotic prescriptions is important because it can lead to more appropriate probiotic use. Furthermore, it is meaningful to determine whether antibiotic-resistant probiotics are often selected by clinicians. Thus, to elucidate the current status of probiotic prescriptions for pediatric patients with otitis media receiving oral AMPC and AMPC/CVA, we performed surveil- 
(A)

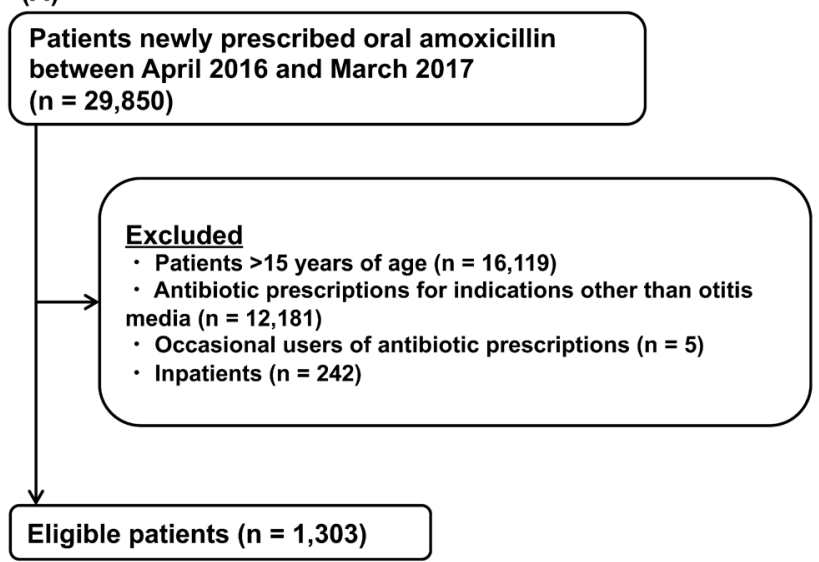

(B)

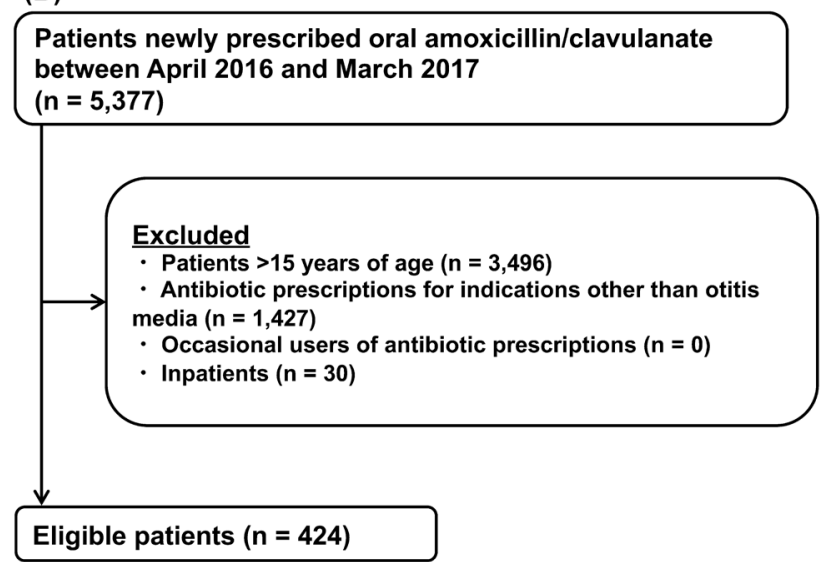

Fig. 1. Flowcharts of Patients Enrolled in the Present Study to Determine the Prescription Status of Probiotics

(A) Flowchart of patients enrolled in the present study who received amoxicillin (AMPC). (B) Flowchart of patients enrolled in the present study who received amoxicillin (AMPC/CVA).

lance using a health insurance claims database.

\section{MATERIALS AND METHODS}

Data Sources For this study we employed data sourced from the JMDC claims database, a Japanese health insurance claims database maintained by the JMDC, Inc. (Tokyo, Japan). ${ }^{19)}$ This database consists of approximately 5.6 million insured individuals and covers approximately $5 \%$ of the population in Japan. This database also includes claims data for company employees and their family members under 75 years of age. ${ }^{19)}$

Study Population We surveyed pediatric patients $(\leq 15$ years of age) who were newly prescribed oral AMPC or AMPC/CVA between April 2016 and March 2017 (the study period) for otitis media. To detect new oral antibiotic prescriptions, we screened data 12 months prior to the study period (the screening period). The exclusion criteria were inpatients and patients who were prescribed occasional antibiotic use. If a single patient was administered multiple rounds of oral AMPC or AMPC/CVA, only the first administration was included to avoid counting the same patient multiple times.

Data Collection AMPC and AMPC/CVA were identified using the Anatomical Therapeutic Chemical (ATC) system, with codes J01CA04 and J01CR02, respectively. Probiotics were identified by the ATC codes A07FA, A07FA01, and A07FA51 and further categorized as antibiotic-resistant probiotics (RLABP or C. butyricum preparations) or non-antibioticresistant probiotics. ${ }^{18)}$ RLABP included Enterococcus faecalis 129 BIO 3B-R of Biofermin R (Biofermin Pharmaceutical Co., Ltd., Kobe, Japan), E. faecalis BIO-4R of Entenoron R and Colepoli R (Meguro Institute Co., Ltd., Osaka, Japan, and Towa Pharmaceutical Co., Ltd., Osaka, Japan), E. faecalis PCR, Lactobacillus acidophilus 4AR, and Bifidobacterium infantis SMR of Lebenin and Lacspan (Wakamoto Pharmaceutical Co., Ltd., Tokyo, Japan, and Kissei Pharmaceutical Co., Ltd., Nagano, Japan), and Bifidobacterium longum of LACB-R (Kowa Company Ltd., Nagoya, Japan). C. butyricum preparations included $C$. butyricum MIYAIRI 588 of MiyaBM (Miyarisan Pharmaceuticals Co., Ltd., Tokyo, Japan), E. faecalis T-110, C. butyricum TO-A, and Bacillus mesentericus TO-A (TOA Biopharma Co., Ltd., Tokyo, Japan).
The infectious disease otitis media was identified according to the diagnostic criteria of the International Classification of Diseases, Tenth Revision (ICD-10) codes of H659, H660, H669 (otitis media and related conditions)].

The clinical departments that prescribed oral antibiotics were identified using text codes. The three clinical departments with the highest frequency of oral antibiotic prescription were collected.

Other patient characteristics collected were age, sex (male/ female), and the duration of oral AMPC, AMPC/CVA, and probiotic administration. We also categorized patients by age ( $\leq 2$ or $>2$ years of age). ${ }^{4)}$ The duration of drug administration was calculated as the total number of prescription days. An interval of more than $3 \mathrm{~d}$ between the two prescriptions was considered to indicate the end of administration.

Outcomes Eligible patients were divided into the AMPC and AMPC/CVA groups. Proportion of probiotic prescriptions was the primary endpoint. In addition, the proportions of antibiotic-resistant probiotic prescriptions were calculated. These outcomes were categorized and assessed by the prescribing clinical department and patient age ( $\leq 2$ or $>2$ years of age). If the probiotics were prescribed on the same day as the antibiotic prescriptions, the case was defined as a "concomitant use of probiotics."

Statistical Analyses Comparison of the categorical variables was performed using Pearson's chi-square or Fisher's exact test. Fisher's exact test was used if more than $20 \%$ of cells had expected frequencies of less than 5 in a $2 \times 2$ contingency table. By using the Shapiro-Wilk normality test and Kolmogorov-Smirnov test, all continuous variables were confirmed to be non-normally distributed. Thus, the MannWhitney $U$ test was used to compare continuous variables. For multiple comparisons, the adjusted $p$-values were calculated using Bonferroni corrections.

A statistically significant difference was defined by an adjusted $p$-value of less than 0.05 . The JMP $14^{\circledR}$ software (SAS Institute, Inc., Cary, NC, U.S.A.) was used for statistical analyses.

Ethics Owing to the anonymity of the data, the institutional review board of the Faculty of Pharmaceutical Sciences of Hokkaido University waived the requirement for informed consent. 
(A)

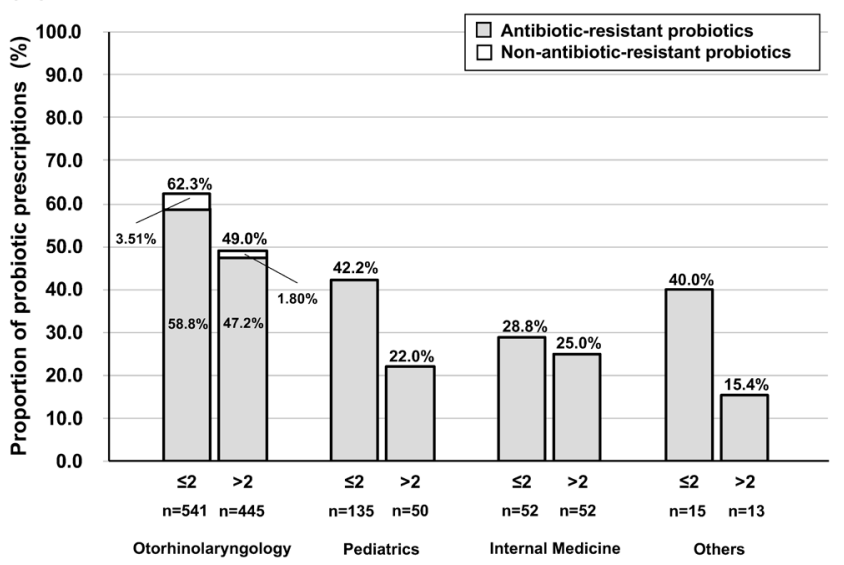

(B)

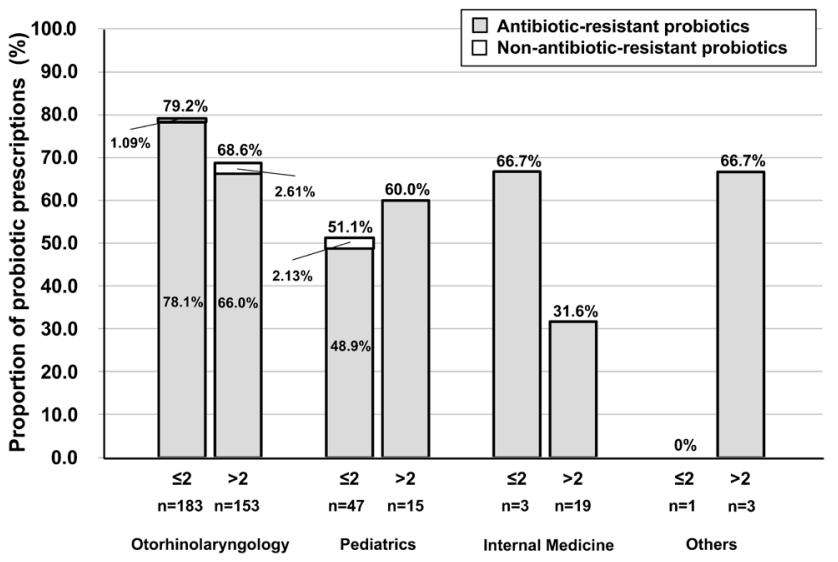

Fig. 2. Prescription Status of Probiotics by $\leq 2$ or $>2$ Years of Age in Each Clinical Department Prescribing Oral Antibiotics

(A) Prescription status of probiotics in patients who received amoxicillin (AMPC). (B) Prescription status of the probiotics in patients who received amoxicillin/clavulanate (AMPC/CVA)." $\leq 2$ " and " $>2$ " indicate $\leq 2$ and $>2$ years of age, respectively.

Table 1. Prescription Status of Probiotics and Characteristics of Patients in the AMPC and AMPC/CVA Group

\begin{tabular}{lccc}
\hline \hline & & \multicolumn{2}{c}{ Total $(n=1303)$} \\
\cline { 2 - 4 } AMPC group & Without probiotics & \multicolumn{2}{c}{ With probiotics $(n=659)$} \\
\cline { 2 - 4 } & $(n=644)$ & $\begin{array}{c}\text { With antibiotic-resistant } \\
\text { probiotics }(n=632)\end{array}$ & $\begin{array}{c}\text { With non-antibiotic-resistant } \\
\text { probiotics }(n=27)\end{array}$ \\
\hline Age (years), median (IQR) & $2(1-6)^{*}$ & $1(0-4)$ & $1(0-5)$ \\
Age (years) $\leq 2$ years of age, $n(\%)$ & $328(50.9)^{*}$ & $396(62.7)$ & $19(70.4)$ \\
Sex (male), $n$ (\%) & $313(48.6)$ & $323(51.1)$ & $6(5-7)$ \\
Duration of antibiotics (d), median (IQR) & $6(5-7)$ & $6(5-8)$ & $5(5-6)$ \\
Duration of probiotics (d), median (IQR) & - & $5(5-6)$ & $22(81.5)$ \\
Equal duration of antibiotics and probiotics, $n(\%)$ & - & $473(74.8)$ & Total $(n=424)$ \\
\hline & & & With probiotics $(n=293)$ \\
AMPC/CVA group & & With antibiotic-resistant & With non-antibiotic-resistant \\
& probiotics $(n=286)$ & probiotics $(n=7)$ \\
\hline Age (years), median (IQR) & $(n=131)$ & $2(0-4)$ & $6(1-12)$ \\
Age (years) $\leq 2$ years of age, $n$ (\%) & & $168(58.7)$ & $3(42.9)$ \\
Sex (male), $n$ (\%) & $3(1-6)$ & $138(48.3)$ & $5(71.4)$ \\
Duration of antibiotics (d), median (IQR) & $63(48.1)$ & $6(5-7)$ & $6(5-10)$ \\
Duration of probiotics (d), median (IQR) & $69(52.7)$ & $5(5-6)$ & $5(4-6)$ \\
Equal duration of antibiotics and probiotics, $n(\%)$ & $6(5-7)$ & $214(74.8)$ & $4(57.1)$ \\
\hline
\end{tabular}

*Adjusted $p$-values $\leq 0.05$ vs. antibiotic-resistant probiotics group, ${ }^{\dagger}$ adjusted $p$-values $\leq 0.05$ vs. non-antibiotic-resistant probiotics group, adjusted $p$-values were calculated using Bonferroni corrections, AMPC: amoxicillin, AMPC/CVA: amoxicillin/clavulanate, IQR: interquartile range.

\section{RESULTS}

The AMPC and AMPC/CVA groups (Figs. 1A, B, Table 1) comprised 1303 and 424 patients, respectively: 50.6\% (659 patients) and 69.1\% (293 patients) were prescribed probiotics, while the proportion of antibiotic-resistant probiotic prescriptions was $95.9 \%$ (632 out of 659 patients) and 97.6\% (286 out of 293 patients), respectively. In addition, 2 patients were prescribed both types of probiotics in the AMPC group, and these patients were included in the antibiotic-resistant probiotics group. On the other hand, there were no patients who received both types of probiotics in the AMPC/CVA group.

Of the patients that received antibiotic-resistant probiotics in the AMPC group, the proportions of RLABP and $C$. butyricum preparations were $89.2 \%$ (564 out of 632 patients) and $11.1 \%$ (70 out of 632 patients), respectively; and 2 patients received both probiotics. In the AMPC/CVA group, the proportions were $88.5 \%$ (253 out of 286 patients) and $11.5 \%$ (33 out of 286 patients), respectively; no patients received both probiotics.

As shown in Table 1, patients without probiotics were generally older than patients who received antibiotic-resistant probiotics in the AMPC group. However, in the AMPC/CVA group, no significant differences were observed.

When classified by the prescribing clinical department and patient age (Figs. 2A, B), patients $>2$ years of age tended to have lower proportions of probiotic prescriptions than patients $\leq 2$ years, except for those in Pediatric and Other departments in the AMPC/CVA group. In both groups, the highest propor- 
tions of probiotic prescriptions were observed in the Otorhinolaryngology department regardless of patient age. Except for patients $>2$ years of age in the Otorhinolaryngology department, the proportion of probiotic prescriptions was less than $50 \%$ in the AMPC group.

\section{DISCUSSION}

We investigated the prescription status of probiotics for pediatric patients with otitis media receiving oral AMPC or AMPC/CVA. The proportion of probiotic prescriptions tended to be higher in the AMPC/CVA group (69.1\%) than in the AMPC group (50.6\%). This may have been due to the broadspectrum action of AMPC/CVA, a risk factor for AAD. ${ }^{8)}$ Notably, most of these patients (95.9 and $97.6 \%$, respectively) were administered antibiotic-resistant probiotics (Table 1). In vitro, probiotics using E. faecalis that were included in RLABP have high minimum inhibitory concentrations (MIC) against ampicillin (the in vitro activity of ampicillin is similar to that of AMPC) ${ }^{18,20)}$ Although C. butyricum has a low MIC against almost all antibiotics, it re-proliferates and exerts its effect when the concentration of antibiotics in the digestive tract becomes low. ${ }^{21)}$ Although the clinical usefulness of "antibiotic-resistant probiotics" has not been completely established relative to "non-antibiotic-resistant probiotics," our results suggest that clinicians make a conscious choice to prescribe "antibiotic-resistant probiotics" when prescribing AMPC and AMPC/CVA.

Patients $>2$ years old tended to obtain lower proportions of probiotic prescriptions in most departments, except for those in Pediatric and Other departments in the AMPC/CVA group (Figs. 2A, B); this may be because older patients have a lower risk of developing $\mathrm{AAD}$ and $\mathrm{C}$. difficile diarrhea. ${ }^{4,7)}$ In addition, the proportions of probiotic prescriptions in the Otorhinolaryngology department were higher than those in the Internal Medicine and Pediatrics departments regardless of age in both groups. Generally, unlike otolaryngologists, internists and pediatricians cover various diseases and may prescribe various antibiotics, such as macrolides, which are often prescribed for respiratory tract infections with a relatively low risk of AAD., ${ }^{3,4)}$ Thus, internists and pediatricians may consider that probiotics are not necessary for the prophylaxis of AAD. Furthermore, our result suggests that Japanese otolaryngologists adhere to the guidelines for the treatment of otitis media; however, this guideline may not be followed by pediatricians and internists owing to differences in specialty. ${ }^{12)}$ Importantly, in the AMPC group, the proportion of probiotic prescriptions was less than $50 \%$, except for patients $>2$ years of age in the Otorhinolaryngology department. In addition, it should be noted that the proportions of probiotic prescriptions are low even in patients $\leq 2$ years old who are at a high risk of AAD and $C$. difficile diarrhea., ${ }^{4,7)}$ Therefore, it will be important to educate clinicians, especially internists and pediatricians, regarding antibiotics and patient backgrounds that are associated with a high risk of AAD in the future.

Based on the national action plan on antimicrobial resistance (AMR), clinicians and pharmacists are required for the appropriate use of antimicrobial agents. ${ }^{22)}$ The occurrence of side effects can lead to cessation of treatment and/or noncompliance, ${ }^{23}$ and as a result may cause not only diminished therapeutic effect, but also the development of AMR. Preven- tion of AAD may contribute to the prevention of AMR. As described above, AMPC and AMPC/CVA are associated with a high risk of $\mathrm{AAD}$, and the preventive effectiveness of probiotics has been established. ${ }^{4,6-9,15)}$ If probiotics are not used concomitantly, it cannot be simply concluded as an "inappropriate prescription."10-12,15) However, considering our results that the proportions of probiotic prescriptions were less than $50 \%$, except for specific populations, clinicians should consider the clinical usefulness of probiotics for children receiving AMPC and AMPC/CVA. ${ }^{10-12,15)}$

Our study had several limitations. First, the JMDC claims database does not include small-sized company employees, self-employed individuals, or their families. ${ }^{19)}$ Thus, the generalizability is unclear. Second, we were unable to evaluate the actual probiotic prescription purposes, that is, whether it was prophylactic for AAD or not. Indeed, the proportions of the equal durations of antibiotics and probiotics were prescribed in $57.1 \%$ and $81.5 \%$ in the AMPC and AMPC/CVA groups, respectively. Third, we did not perform a thorough evaluation of the accuracy of the diagnosis (i.e., their ICD-10 codes) recorded in the claims database.

In conclusion, our results indicated the probability of insufficient probiotic prescriptions in pediatric patients with otitis media, except for specific populations in Japan. Solving this issue may lead to more safe antimicrobial therapy.

Conflict of Interest The authors declare no conflict of interests.

\section{REFERENCES}

1) McFarland LV. Antibiotic-associated diarrhea: epidemiology, trends and treatment. Future Microbiol., 3, 563-578 (2008).

2) Gogate A, De A, Nanivadekar R, Mathur M, Saraswathi K, Jog A, Kulkarni MV. Diagnostic role of stool culture \& toxin detection in antibiotic associated diarrhoea due to Clostridium difficile in children. Indian J. Med. Res., 122, 518-524 (2005).

3) Uda K, Okubo Y, Kinoshita N, Morisaki N, Kasai M, Horikoshi Y, Miyairi I. Nationwide survey of indications for oral antimicrobial prescription for pediatric patients from 2013 to 2016 in Japan. $J$. Infect. Chemother., 25, 758-763 (2019).

4) Turck D, Bernet JP, Marx J, Kempf H, Giard P, Walbaum O, Lacombe A, Rembert F, Toursel F, Bernasconi P, Gottrand F, McFarland LV, Bloch $\mathrm{K}$. Incidence and risk factors of oral antibioticassociated diarrhea in an outpatient pediatric population. J. Pediatr. Gastroenterol. Nutr, 37, 22-26 (2003).

5) Elstner CL, Lindsay AN, Book LS, Matsen JM. Lack of relationship of Clostridium difficile to antibiotic-associated diarrhea in children. Pediatr. Infect. Dis., 2, 364-366 (1983).

6) Kramer MS, Hutchinson TA, Naimark L, Contardi R, Flegel KM, Leduc DG. Antibiotic-associated gastrointestinal symptoms in general pediatric outpatients. Pediatrics, 76, 365-370 (1985).

7) Wiström J, Norrby SR, Myhre EB, Eriksson S, Granström G, Lagergren L, Englund G, Nord CE, Svenungsson B. Frequency of antibiotic-associated diarrhoea in 2462 antibiotic-treated hospitalized patients: a prospective study. J. Antimicrob. Chemother., 47, 43-50 (2001).

8) Agamennone V, Krul CAM, Rijkers G, Kort R. A practical guide for probiotics applied to the case of antibiotic-associated diarrhea in The Netherlands. BMC Gastroenterol., 18, 103 (2018).

9) Hum SW, Shaikh KJ, Musa SS, Shaikh N. Adverse events of antibiotics used to treat acute otitis media in children: a systematic metaanalysis. J. Pediatr., 215, 139-143.e7 (2019). 
10) Chow AW, Benninger MS, Brook I, Brozek JL, Goldstein EJ, Hicks LA, Pankey GA, Seleznick M, Volturo G, Wald ER, File TM Jr. IDSA clinical practice guideline for acute bacterial rhinosinusitis in children and adults. Clin. Infect. Dis., 54, e72-e112 (2012).

11) Wald ER, Applegate KE, Bordley C, Darrow DH, Glode MP, Marcy SM, Nelson CE, Rosenfeld RM, Shaikh N, Smith MJ, Williams PV, Weinberg ST. Clinical practice guideline for the diagnosis and management of acute bacterial sinusitis in children aged 1 to 18 years. Pediatrics, 132, e262-e280 (2013)

12) Japan Otological Society, Japan Society for Pediatric ORL, Japan Society for Infection and Aerosol in Otorhinolaryngology. Clinical Practice Guidelines for the Diagnosis and Management of Acute Otitis Media (AOM) in Children in Japan, pp. 80-82 (2018).

13) Ohnishi K, Ainoda Y, Imamura A, Iwabuchi S, Okuda M, Nakano T. JAID/JSC Guidelines for Infection Treatment 2015-Intestinal infections. J. Infect. Chemother., 24, 1-17 (2018).

14) Fuller R. Probiotics in human medicine. Gut, 32, 439-442 (1991).

15) Guo Q, Goldenberg JZ, Humphrey C, El Dib R, Johnston BC. Probiotics for the prevention of pediatric antibiotic-associated diarrhea. Cochrane Database Syst. Rev., 4, CD004827 (2019).

16) Seki H, Shiohara M, Matsumura T, Miyagawa N, Tanaka M, Komiyama A, Kurata S. Prevention of antibiotic-associated diarrhea in children by Clostridium butyricum MIYAIRI. Pediatr. Int., $\mathbf{4 5}$,
86-90 (2003).

17) Egashira K, Kitahara T, Kashiwagi K, Higuchi N, Nakashima M, Ichikawa N, Sasaki H. Investigation for proper use of probiotics in Nagasaki University Hospital of Medicine and Dentistry. Yakugaku Zasshi, 126, 1155-1161 (2006).

18) Yamaguchi T, Miura Y, Matsumoto T. Antimicrobial susceptibility of Enterococcus strains used in clinical practice as probiotics. $J$. Infect. Chemother., 19, 1109-1115 (2013).

19) Japan Medical Data Center. 〈https://www.jmdc.co.jp/en/», accessed 2 February, 2020.

20) Malik ZA, Litman N. Ampicillin and Amoxicillin. Pediatr. Rev., 27, 434-436 (2006).

21) Kaneko N, Nakayama T, Ichikawa N. Susceptibility of spore-forming butyric acid bacteria to antimicrobial agents. Yakugaku Zasshi, 132, 849-853 (2012).

22) The Government of Japan. "National action plan on antimicrobial resistance (AMR).”: 〈https://www.mhlw.go.jp/file/06-Seisakujouhou10900000-Kenkoukyoku/0000138942.pdf), accessed 29 October, 2020.

23) Lambert M, Conus P, Eide P, Mass R, Karow A, Moritz S, Golks D, Naber D. Impact of present and past antipsychotic side effects on attitude toward typical antipsychotic treatment and adherence. Eur. Psychiatry, 19, 415-422 (2004). 